Soziologie und Ökonomie: Der Beitrag experimenteller Wirtschaftsforschung zur Sozialtheorie*

\author{
Journal Article \\ Author(s): \\ Diekmann, Andreas \\ Publication date: \\ 2008
}

Permanent link:

https://doi.org/10.3929/ethz-b-000014798

Rights / license:

In Copyright - Non-Commercial Use Permitted

Originally published in:

Kölner Zeitschrift für Soziologie und Sozialpsychologie 60(3), https://doi.org/10.1007/s11577-008-0026-5 


\title{
Soziologie und Ökonomie: Der Beitrag experimenteller Wirtschaftsforschung zur Sozialtheorie*
}

\author{
Andreas Diekmann
}

Zusammenfassung: Der Artikel diskutiert Befunde aus der experimentellen Wirtschaftsforschung, die an klassischen soziologischen Erkenntnissen anknüpfen. Insbesondere werden Studien zum Altruismus, über Reziprozität, soziale Normen und Sanktionen behandelt und der Beitrag dieser Studien zur Weiterentwicklung der Sozialtheorie herausgearbeitet. Darüber hinaus werden methodische Aspekte angesprochen und es wird dafür plädiert, dass die Soziologie sich wieder darauf besinnen sollte, dass zur Methodenvielfalt auch Experimente und Feldexperimente gehören. Ökonomen auf der anderen Seite sollten den interdisziplinären Charakter des Forschungsprogramms stärker betonen, an dem Forscherinnen und Forscher verschiedener Disziplinen beteiligt sind.

Stichworte: Experimentelle Spieltheorie · Experimentelle Wirtschaftsforschung - Reziprozität · Altruismus · Soziale Normen und Sanktionen · Soziale Kooperation

\begin{abstract}
Almost unnoticed by sociologists, experimental economics has expanded its research to many classical sociological topics such as the evolution and stability of social norms, social sanctions and social order, reciprocity, and pro-social behaviour. This article reports the main empirical findings and theories, their strengths and weaknesses, and explores how experimental economics has furthered social theory. It concludes with the suggestion that sociology should bring experimental methods back into the field and that economics should acknowledge that the research program is an interdisciplinary task analyzing the basic aspects of human behaviour.
\end{abstract}

Keywords: Experimental Game-theory · Experimental Economic-research • Reciprocity · Altruism · Social Norms and Sanctions · Social Cooperation

In der experimentellen Wirtschaftsforschung ist seit rund zwei Jahrzehnten eine wachsende Forschungsaktivität zu beobachten, die sich neben ökonomischen Fragen im engeren Sinne auf klassische Themen der Soziologie richtet. Gemeint ist nicht die Aus-

Andreas Diekmann ( $\square)$

ETH Zürich, Soziologie / SEW E 26, Scheuchzerstraße 68/70, 8092 Zürich, Schweiz

E-Mail: andreas.diekmann@soz.gess.ethz.ch

* Ich bedanke mich für Hinweise und Anregungen bei Manuela Vieth und dem anonymen Verfasser oder der Verfasserin eines Gutachtens zu diesem Manuskript. 
weitung ökonomischer Methoden auf traditionell von Soziologen bearbeitete Gebiete wie z. B. bei der Familienökonomie Gary Beckers (1981). Von der Soziologie fast unbemerkt geht es vielmehr um soziologische Schlüsselprobleme, um Grundlagen der Sozialtheorie. „Wirtschaftsforschung“ ist dafür ein irreführender Begriff, denn im Mittelpunkt stehen Themen wie Normen und Sanktionen, Reziprozität, Altruismus und Moral und das seit Thomas Hobbes in der Sozialtheorie zentrale Problem sozialer Ordnung. Es ist schon erstaunlich, dass in der Soziologie von diesen Entwicklungen kaum Kenntnis genommen wird. Die Soziologie wäre gut beraten, Ergebnisse der experimentellen Ökonomie aufzunehmen und in einen Dialog einzutreten. Die Ökonomen auf der anderen Seite sollten sich daran erinnern, dass sie auch an Theorien und Hypothesen aus soziologischer Forschung anknüpfen und auf Ergebnissen der Sozialpsychologie aufbauen.

In diesem Artikel wird zunächst die Entwicklung der experimentellen Wirtschaftsforschung skizziert. In Abschnitt 2 zwei werden wichtige Befunde herausgearbeitet und im dritten Abschnitt erklärende Modelle diskutiert. Abschließend wird die Bedeutung des Forschungsprogramms für die Soziologie angesprochen.

\section{Der Hintergrund: Neue Entwicklungen in der Ökonomie}

Vorurteile sind nicht nur zwischen den Bevölkerungen benachbarter Länder, sondern auch zwischen benachbarten Disziplinen zählebig. Die Kritik am „Modellplatonismus“ der Ökonomie hatte einen berechtigten Kern, auf die empirische Wirtschaftsforschung trifft sie heute allerdings nicht mehr zu (vgl. auch Albert 1965, 2008). Zwei miteinander eng verknüpfte Entwicklungen haben der empirischen und speziell der experimentellen Wirtschaftsforschung den Boden bereitet: Die „mikroökonomische Wende“ in der Ökonomie und damit eng verbunden der Aufstieg der Spieltheorie seit Beginn der 1980er Jahre. Mit der Mikroökonomie kamen das Interesse an Modellen strategischer Interdependenz von Akteuren, d. h. der Spieltheorie, und das Interesse an der Erhebung von Mikro- oder Individualdaten. Nicht zufällig wurde Anfang der 1980er Jahre das sozioökonomische Panel aus der Taufe gehoben. In dieser Zeit wuchs der Bedarf an Mikrodaten. Die Surveyforschung, sonst eine Domäne der Soziologie, hielt vermehrt Einzug im Bereich der Wirtschaftsforschung, die Ökonomie wurde insgesamt empirischer. Mikroökonomie, der Bedarf an empirischen Daten und Weiterentwicklungen in der Spieltheorie bildeten eine Gemengelage, die die aufstrebende experimentelle Wirtschaftsforschung vorantrieb. Vergessen wird leicht, dass der Boden durch die experimentelle Spieltheorie seit den 1950er Jahren bereitet wurde. Lange vor dem Aufschwung der experimentellen Wirtschaftsforschung wurden allein mit dem Gefangenendilemma hunderte von Studien, meist unter Federführung von Sozialpsychologen, publiziert. Immer noch häufig zitiert wird die Pionierarbeit von Rapoport und Chammah (1965) (siehe auch Rapoport 1998) über das wiederholte Gefangenendilemma und den Erfolg der Tit-for-Tat-Strategie, später Gewinner des von Axelrod (1987) arrangierten Computerturniers zur „Evolution der Kooperation“. Die experimentelle Methode war dagegen vor Mitte der 1980er Jahre in der Ökonomie nahezu unbekannt. Reinhard Selten und Heinz Sauermann waren Außenseiter, als sie vor gut einem halben Jahrhundert erste Anstöße für die experimentelle Wirtschaftsforschung in Deutschland gegeben hatten. Auch die 
Spieltheorie fristete in der Ökonomie lange ein Kümmerdasein. Das vom Nobelpreiskomitee 1994 u. a. ausgezeichnete Konzept der „Teilspielperfektheit“" wurde zunächst auf wenigen Seiten, verborgen in einer Arbeit über Oligopole, in einer deutschsprachigen Fachzeitschrift veröffentlicht (Selten 1965). In den vergangenen zwei oder drei Jahrzehnten zeichnete sich nicht nur eine vermehrte Rezeption der Spieltheorie in der Ökonomie ab. Hinzu kamen zahlreiche Innovationen. Mit wiederholten Spielen, unvollkommener Information, Informationsasymmetrien, Signalspielen und der evolutionären Spieltheorie konnte der Anwendungsbereich der Spieltheorie stark erweitert werden. Erkannt wurde freilich auch, dass die Verhaltensannahmen für deskriptive Erklärungen häufig zu restriktiv waren. Kognitive Psychologie und Entscheidungsforschung (Tversky/Kahneman 1981) beunruhigten die Ökonomie mit immer neuen „Anomalien“ (Thaler 1992). Diese Forschungsstränge wurden von der experimentellen Wirtschaftsforschung aufgegriffen und mündeten in das Forschungsprogramm der „Behavioral Game Theory“ (Camerer 1997, 2003). Methoden, die in anderen Disziplinen seit langer Zeit einen festen Platz hatten, wurden nun auch in der Ökonomie verwendet oder quasi ,neu erfunden".

In der Soziologie wurden früher vermutlich mehr experimentelle Studien durchgeführt als heute, während es sich in der Ökonomie genau umgekehrt verhält ${ }^{1}$. Dabei ist die experimentelle Methode mit randomisierten Versuchsgruppen, sofern anwendbar, immer noch der beste Weg, um kausalen Zusammenhängen auf die Spur zu kommen. Das typische Experiment der „Wirtschaftsforscher" findet im Labor mit Studenten als Versuchspersonen statt. Im Labor stehen miteinander vernetzte Computer. Instruktionen und Entscheidungssituationen erscheinen auf dem Bildschirm. Zur Programmierung existiert flexible Software, wie z. B. das von Fischbacher (2006) entwickelte Programm „z-tree“. Anders als in vielen psychologischen Experimenten wird in der Regel auf die Täuschung von Versuchspersonen verzichtet (aber auch mit gewissen Ausnahmen; zu einer Diskussion siehe Weimann 1994). Weiterhin werden die Versuchspersonen in der Regel mit Geldbeträgen oder Punkten, die nach Abschluss des Experiments in Geldbeträge konvertiert werden können, erfolgsabhängig entlohnt. Eine künstliche Laborsituation, die der Komplexität sozialen Geschehens nicht gerecht wird, könnte man einwenden. Dabei ist aber zu bedenken, dass Experimente nicht der Aufdeckung von Zusammenhängen, sondern primär der Prüfung von Kausalhypothesen dienen. Berücksichtigt man die entscheidenden Variablen und ihre Interaktionen, kann man auch aus Laborexperimenten sehr viel über die grundlegenden Muster sozialen Verhaltens lernen. Experimente sind quasi „Wahrheitsfilter“, die aus einer Menge von theoretisch inspirierten Hypothesen die korrekten Hypothesen heraussortieren sollen. Es ist bekannt, dass dies nicht immer unproblematisch ist und zahlreiche Fehlerquellen existieren. Skepsis gegenüber der Künstlichkeit von Laborexperimenten kann durch Feldexperimente im „,natürlichen“

1 Eine Inhaltsanalyse englischsprachiger Fachzeitschriften bestätigt die Einschätzung für die heutige Situation. Im Zeitraum 2005 bis 2007 beträgt der Anteil von Artikeln, die Ergebnisse auf der Basis experimenteller Methoden berichten, 0 Prozent im British Journal of Sociology (0 von 75 Artikeln), 2,7 Prozent im American Journal of Sociology (3/111) und 0,8 Prozent im American Sociological Review (1/126). Dagegen sind es im American Economic Review 12,2 Prozent (33/270). Für die Durchführung der Inhaltsanalyse bedanke ich mich bei Matthias Näf. 
sozialen Umfeld, durch die Analyse nicht-reaktiver Verhaltensdaten und durch ergänzende Surveystudien begegnet werden. Solche Kreuzvalidierungen, in zunehmendem Masse auch mit Feldexperimenten, finden auch in der experimentellen Wirtschaftsforschung statt. Feldexperimente und die Analyse nicht-reaktiver „Verhaltensspuren“ wurden bereits vor einigen Jahrzehnten in der Soziologie und Sozialpsychologie unter dem Stichwort „Unobtrusive Measures“ (Webb et al. 1966; Friedrichs 1973; Bungart/Lück 1974) propagiert. Heute zählen diese Methoden zum Repertoire experimenteller Wirtschaftsforschung (Harrison/List 2004). Sie werden z. B. eingesetzt, um die Diskriminierung von Bevölkerungsgruppen, Probleme der Kooperation oder prosoziales Verhalten zu untersuchen. Beispiele werden wir im folgenden Abschnitt behandeln. ${ }^{2}$

\section{Ergebnisse experimenteller Wirtschaftsforschung}

Wir betrachten Ergebnisse aus drei miteinander verbundenen Themenkreisen, die für die Sozialtheorie von grundlegender Bedeutung sind: Altruismus, Reziprozität und soziale Normen. Dabei werden auch Experimente zur Sprache kommen, die nicht von Wirtschaftsforschern stammen, sondern u. a. aus Psychologie und Soziologie, aber zu diesem Themenkreis gehören. Wie erwähnt ist das Forschungsprogramm interdisziplinär. Die drei Themen bauen aufeinander auf. Reziprozität in einmaligen oder endlich wiederholten Situationen ist ein Spezialfall altruistischen Verhaltens. Altruistische Reziprozität ist wiederum bedeutsam für die Stabilität von Normen und Sanktionen und trägt damit entscheidend zum Zusammenhalt der Gesellschaft bei.

\subsection{Altruismus und prosoziales Verhalten: „Der brave Mann denkt an sich selbst zu- letzt"}

„Denkt der brave Mann“ in Friedrich Schillers Wilhelm Tell an „sich selbst zuletzt“ oder müssen wir vor dem Wort „selbst“ ein Komma einsetzen? Ohne Komma „Altruismus“, mit Komma „homo oeconomicus“. Wie Menschen sich wirklich verhalten, darüber geben auch Experimente Auskunft. „Altruismus“ wird in der experimentellen Wirtschaftsforschung definiert als „Kosten verursachende Handlungen, die anderen Personen ökonomische Vorteile verschaffen“ (Fehr/Fischbacher 2003). „Ökonomische Vorteile“ sind nicht notwendigerweise materiell. Pflege, Hilfe und andere Dienste zählen dazu. Diese Definition verweist nicht auf Motive, so dass tautologische Fallstricke vermieden werden.

Kooperatives Verhalten ist nicht per se altruistisch. Im unendlich oft wiederholten Gefangenendilemma mit genügend großem Wert der Zukunft werden sich Akteure im Eigeninteresse für (bedingt) kooperative Strategien entscheiden (Taylor 1976; Axelrod 1987).

2 Viele der im Folgenden zitierten Arbeiten findet man in dem Archiv „Research Papers in Economics“ (REPEC) im WWW. Das REPEC-Archiv enthält mehr als 230000 Arbeitspapiere, die zum Download frei zugänglich sind, sowie über 340000 Hinweise und Abstracts von Fachartikeln. Der Aufbau eines Archivs mit soziologischen Arbeiten nach diesem Vorbild wäre nur zu empfehlen. 
Das Kriterium der „Kosten verursachenden Handlungen“ ist hier nicht gegeben, auch wenn die Kooperation den Mit-Akteuren Vorteile verschafft. Eine Anekdote erzählt von einem kleinen walisischen Dorf, das oft von Touristen besucht wird. Es hat sich herumgesprochen, dass in dem Dorf ein Narr lebt, der bei der Wahl zwischen einer Pfundnote und einem Schilling die Münze vorzieht. Immer wieder kommen Touristen, stellen ihn vor die Wahl zwischen Pfundnote und Schilling und freuen sich, wenn der „Narr“ nach der Münze greift. Einmal der Verlockung nach dem Geldschein nachgeben und das Geschäftsprinzip wäre dahin (Gigerenzer 2000).

Anders verhält es sich in einmaligen Entscheidungssituationen. Seit langem ist bekannt, dass sich ein erheblicher Anteil von Personen in einmaligen und endlich oft wiederholten Dilemma-Situationen oder „Public-Good-Games“ kooperativ verhält und zum Kollektivgut beiträgt, obwohl unter der Annahme einer eigeninteressierten, individuellrationalen Nash-Gleichgewichtsstrategie nicht-kooperatives Verhalten zu erwarten wäre. Im Public-Good-Game z. B. können Personen einen Teil oder ihren gesamten Einsatz in einen Fonds einzahlen. Der Betrag im Fonds wird vom Versuchsleiter beispielsweise um 50 Prozent erhöht und gleich unter alle Teilnehmer aufgeteilt. Vollständige Kooperation ist zwar pareto-optimal, jedoch haben alle Teilnehmer einen Anreiz zu defektieren und nicht in den kollektiven Fonds einzuzahlen. Experimente zeigen, dass ein Teil der Personen kooperiert, d. h. nicht die rational-eigennützige Strategie wählt (Überblick in Ledyard 1995). Paradigma experimenteller Altruismusforschung ist das „Diktatorspiel“, bei dem eine Versuchsperson in der Rolle des Diktators einen gegebenen Betrag auf sich und den Mitspieler aufteilen soll. Streng genommen handelt es sich nicht um ein „Spiel“, sondern um eine einfache Entscheidungssituation, bei der das Ergebnis nur vom Verhalten eines Akteurs abhängt und strategisches Verhalten nicht involviert ist. Zahlreiche Experimente mit dem Diktatorspiel liefern Belege für altruistisches Verhalten. Selbst bei relativ hohen Auszahlungen und unter Wahrung von Anonymität geben die meisten Personen einen Teil des Betrags bis maximal 50 Prozent an einen anonym bleibenden Mitspieler ab. Auch Kinder verhalten sich im Diktatorspiel altruistisch, wobei die Abgabequote mit dem Alter und dem Sozialstatus der Eltern ansteigt (Benenson et al. 2007). Sozialisation unterstützt offenbar das Ausmaß an Altruismus. Andreoni und Miller (2002) können in einem Experiment mit einer Serie von Diktatorspielen zeigen, dass sich Personen konsistent altruistisch und rational verhalten (d. h. die altruistischen Entscheidungen stehen unter Preis- und Budgetrestriktionen im Einklang mit den Axiomen der Theorie der ,revealed preferences“). Weiterhin sprechen Experimente mit sequentiellen Spielen, die Entscheidungen der Akteure erfolgen hier zeitlich nacheinander und der nachfolgend handelnde Akteur ist über die vorhergehenden Entscheidungen informiert, für altruistische Reziprozität (Fehr/Fischbacher 2003). Diesen wichtigen Spezialfall altruistischer Handlungen mit weit reichenden Konsequenzen für Soziologie und Sozialtheorie werden wir weiter unten noch genauer betrachten. Auch eine öffentlich gemachte Selbstverpflichtung kann Kosten verursachendes, kooperatives Verhalten hervorrufen. In einem Experiment von Prosch (2007) konnten Versuchspersonen vor einer Entscheidung in einem Chickenspiel ein Ehrenwort abgeben, dass sie sich kooperativ verhalten werden. Eine sehr große Mehrheit der Personen gab ihr Ehrenwort und hielt sich daran, ohne dass bei Verletzung des Versprechens irgendwelche Sanktionen vorgesehen waren. 
In der Sozialpsychologie existiert eine Vielzahl von Untersuchungen über Hilfeleistungen, Spenden oder allgemeiner über prosoziales Verhalten. Prosoziales Verhalten gegenüber Fremden in einmaligen Situationen ist ein Beleg für Altruismus, sofern Dritte nicht davon erfahren und wie bei anonymen Spenden keine Reputationseffekte beteiligt sind. Prosoziales Verhalten wird aber auch durch andere Faktoren begünstigt oder gehemmt. Die bekannte Untersuchung von Darley und Latanè (1968) macht auf die Bedeutung der „Verantwortungsdiffusion“ aufmerksam: Je mehr potentiell Hilfe leistende Personen wahrgenommen werden, desto geringer ist die eigene Bereitschaft zur Hilfeleistung. Strategische Interaktion kann die Wahrscheinlichkeit einer Hilfeleistung beeinflussen. Spieltheoretisch kann diese Situation als „Volunteer's Dilemma“ formalisiert werden (Diekmann 1985, 1993). Verschiedene, empirisch prüfbare Hypothesen sind aus dem Modell ableitbar, insbesondere die Hypothese der Verantwortungsdiffusion, die genau mit der Nash-Gleichgewichtsstrategie übereinstimmt. Wie bei anderen sozialen Dilemmata ist das Ergebnis der Verantwortungsdiffusion nicht pareto-optimal. Anknüpfend an diese Überlegungen haben die Ökonomen Barron und Yechiam (2002) den Einfluss der Verantwortungsdiffusion auf das Ausmaß altruistischen und prosozialen Verhaltens in einem einfachen, aber pfiffig arrangierten Feldexperiment untersucht. Das Design sah 240 E-Mail-Anfragen einer fiktiven Sarah Feldman vor, die in der Kontrollbedingung an einzelne Empfänger und in der Versuchsbedingung, in den EMail-Kopfzeilen gut sichtbar, an Gruppen von Empfängern gerichtet waren. Bei einem einzelnen Empfänger lag die Auskunftshäufigkeit bei 64 Prozent, bei fünf Empfängern waren es 50 Prozent. Auch die durchschnittliche Länge hilfreicher Antworten, gemessen als Zahl der Worte, war signifikant größer bei einem einzelnen Empfänger gegenüber E-Mails von Empfängern in der Fünf-Personen-Gruppe.

Ökonomen bauen auf den Forschungen zum prosozialen Verhalten auf, um z. B. das Ausmaß des Beitrags zu kollektiven Gütern oder paradoxe Preiseffekte zu erklären (Überblick in Meier 2006). Titmuss (1970) hat bereits darauf hingewiesen, dass die Ersetzung freiwilliger durch bezahlte Blutspenden zu einem Rückgang des Spendenverhaltens führen könnte. ${ }^{3}$ Ganz im Gegensatz zur ökonomischen Logik hat ein zusätzlicher monetärer Anreiz nicht immer das erwünschte Verhalten zur Folge, denn monetäre Anreize können unter bestimmten Bedingungen die intrinsische Motivation zu altruistischem Handeln verdrängen (Deci 1975; Frey 1997; Frey/Jegen 2001). Ein bemerkenswertes Beispiel für die Verdrängung (,crowding out") intrinsischer Motivation lieferte ein Feldexperiment in israelischen Kindergärten (Gneezy/Rustichini 2000a; Häring/Storbeck 2007). Wie überall in der Welt, mussten die Eltern ihre Kinder bis zu einem bestimmten Zeitpunkt abholen. Wurde der Zeitpunkt überschritten, musste das Personal warten. Gneezy und Rustichini untersuchten in ihrem Experiment, ob die Einführung einer Strafgebühr einen Einfluß auf das Verhalten der Eltern hat. In sechs von zehn Kindergärten wurde eine Gebühr für das späte Abholen der Kinder (mehr als 10

3 Goette und Stutzer (2008) haben die Titmuss-These anhand von mehr als 10000 Schweizer Blutspendern überprüft. Im Gegensatz zu der Hypothese erhöhte ein materieller Anreiz (ein Lotterieticket im Wert von 5 CHF) die Bereitschaft zum Blutspenden. Allerdings trat der Effekt nur bei Personen auf, die seltener Blut spendeten. Bei den häufigen, innerlich besonders überzeugten Blutspendern hatte die materielle Belohnung keinen Effekt. 
Minuten nach der vereinbarten Zeit) verlangt, die übrigen vier Kindergärten bildeten die Kontrollgruppe. Statt eines Rückgangs hatte die neue Regelung eine Zunahme der Überstunden des Personals zur Konsequenz. In den sechs Kindergärten mit Strafzahlung stieg der Anteil der zu spät kommenden Eltern signifikant an, während in der Kontrollgruppe kein Effekt zu verzeichnen war. Eine Erklärung dafür ist, dass die Eltern nun kein schlechtes Gewissen mehr hatten, wenn sie ihre Kinder später abholten, denn sie bezahlten ja für die Leistung des Kindergartens. Aber mehr noch: Nach Abschaffung der Gebühr in einer späteren Phase des Experiments blieb der Anteil auf dem erhöhten Niveau. Die intrinsische Motivation, die Norm einzuhalten, wurde durch den finanziellen Anreiz verdrängt. In einfallsreichen Experimenten wurden weitere Belege für das „,crowding out“ intrinsischer Motivation gefunden, allerdings sind die Zusammenhänge komplexer und nicht linear. So zeigte sich in einem Experiment, bei dem Schulkinder Spenden für karitative Zwecke sammelten, dass insbesondere geringe finanzielle Anreize zu einem starken Verdrängungseffekt führten. Das Resultat hat auch Konsequenzen für die Entschädigung von Versuchspersonen in Experimenten. $\mathrm{Zu}$ geringe monetäre Anreize können die Ergebnisse möglicherweise stärker verzerren als Experimente ohne finanzielle Anreize. „Pay enough or don't pay at all“ lautet der Titel der Studie (Gneezy/Rustichini 2000b).

Fallstudien und Feldexperimente weisen den Vorteil der Nicht-Reaktivität auf. Ein Versuchsleiter-Effekt, der in Laborexperimenten zu Verzerrungen führen könnte, existiert hier nicht. Keiner der 240 Empfänger des oben beschriebenen E-Mail-Experiments wusste, dass es sich um ein Experiment handelte. Solange es um alltägliche Handlungen mit relativ geringem Aufwand geht, dürften solche Experimente mit „unfreiwilligen“ Teilnehmern auch ethisch vertretbar sein.

Wie valide sind die Ergebnisse von Laborexperimenten altruistischen Verhaltens? Benz und Meier (2006) haben Zusammenhänge zwischen der Spendenbereitschaft und den Entscheidungen in einem Laborexperiment untersucht. Die für ihre Studie zugängliche Studentenkartei gab Auskunft darüber, ob Studierende zu Beginn eines Semesters freiwillig eine Spende für Studierende mit finanziellen Schwierigkeiten oder für die Unterstützung ausländischer Studenten oder für beide Sozialfonds gegeben hatten. Die Daten gaben über das Verhalten für mehrere Semester vor und nach den Experimenten Auskunft. In zwei Experimenten wurden ähnliche Entscheidungssituationen simuliert. In Entscheidungssituation eins konnten die Versuchspersonen in die oben erwähnten Sozialfonds der Universität einzahlen, in Experiment zwei bezog sich die Entscheidung auf allgemeine Wohltätigkeitsfonds. Die Korrelation zwischen den Spenden im Sozialfonds- Experiment und dem Durchschnitt der Spenden in vier Semestern vor dem Experiment betrug 0,28, die Korrelation mit der Spendenhöhe in den vier Semestern nach dem Experiment 0,40. Beim Wohltätigkeitsfonds waren die Korrelationen mit 0,26 bzw. 0,25, wie erwartet, etwas niedriger. Wenn man bedenkt, so argumentieren die Autoren, dass altruistisches Verhalten auch stark von situativen Faktoren abhängig ist, sind die Korrelationen als relativ hoch einzustufen. Immerhin zeigen die Ergebnisse, dass Entscheidungen in der künstlichen Laborwelt durchaus mit dem Verhalten in der ,realen Außenwelt" korreliert sind. Kritischer sind die aufgrund einer Studie von Cherry, Frykblom und Shogren (2002) formulierbaren Einwände gegenüber dem hohen Ausmaß von Altruismus in Experimenten. Selbst verdiente Einsätze statt „windfall profits“ und 
strikte Anonymität lauten die beiden Stichworte. Die Versuchspersonen bekommen in der Regel in Geld umtauschbare Spielpunkte als Anfangsausstattung geschenkt. Die Vermutung ist begründet, dass man bei solchen „windfall profits“ eher großzügiger ist als bei selbst erarbeiteten Einkommen. Zudem wird ein Experiment, bei dem die individuellen Entscheidungen absolut anonym sind und vor allem von den Versuchspersonen als anonym wahrgenommen werden, weniger altruistische Entscheidungen hervorbringen. Kooperation, Fairness und ein gewisses Maß an Altruismus sind sozial erwünschte Verhaltensweisen. Wer geht schon gerne nach dem Diktatorspiel als bekannt gieriger Raffke aus dem Labor? Selbst bei weitgehender Anonymisierung kann bei computerunterstützten Experimenten immer noch ein Restverdacht bleiben, dass der Versuchsleiter die Teilnehmer identifizieren könnte. Mit der Devise „hardnose the dictator“ wählen Cherry et al. die „Drosophila“ der Altruismusforschung, nämlich das Diktatorspiel, als Testfall. In der Kontrollgruppe erfolgt die Durchführung ähnlich wie in anderen Experimenten. Für den „Diktator“ bleibt der Mitspieler anonym und umgekehrt ebenso, der Versuchsleiter aber kennt die Aufteilung. Die Anfangsausstattung wird vom Versuchsleiter zur Verfügung gestellt. In Versuchsgruppe eins wird die Anfangsausstattung dagegen mit einem Quiz verdient. In Versuchsgruppe zwei wird das Experiment zusätzlich „,doppelblind“ und strikt anonym durchgeführt. Abgeschirmt von anderen Versuchspersonen und dem Versuchsleiter teilen die Teilnehmer in der Rolle des Diktators den Geldbetrag auf, legen das Geld in einen Umschlag, verschließen den Umschlag und werfen ihn in einen Kasten. In den drei Gruppen wird zusätzlich der aufzuteilende Geldbetrag variiert. In der Low-Stake-Bedingung sind es 10 US-Dollar, in der High-Stake-Bedingung 40 Dollar. In der Kontrollgruppe gaben 81 Prozent (low stake) und 85 Prozent (high stake) einen Geldbetrag an den Mitspieler ab. Wurde der Einsatz selbst verdient, betrug der Anteil altruistischer Akteure nurmehr 21 bzw. 30 Prozent. In der Doppelblind-Bedingung (plus selbst verdienter Einsatz) war nur noch ein Anteil altruistischer Aufteilungen von 5 Prozent bzw. 3 Prozent zu beobachten. Sollte sich das Ergebnis der Studie von Cherry et al. als robust und replizierbar herausstellen, dann dürfte es sich bei den bisher in zahlreichen Diktator-Experimenten gefundenen Ergebnissen über altruistisches Verhalten um nach oben verzerrte Schätzungen handeln. Allerdings ist auch die Verteilung von „windfall profits“ an andere Personen altruistisches Verhalten. Das Experiment liefert die Erkenntnis, dass verdiente Einkommen das Ausmaß der Abgabebereitschaft stark verringern. Es beweist aber nicht, dass Altruismus im Diktator-Spiel nur ein methodisches Artefakt sei. Lediglich die Reduktion von Altruismus, die auf die zweite Bedingung des Doppelblindversuchs zurückgeht, kann als methodisches Artefakt gelten. Dass „windfall profits“ die Spendierfreudigkeit erhöhen, könnte man auch als speziellen Fall von reziprokem Altruismus betrachten.

Insgesamt zeigen Experimente mit sozialen Dilemmata, mit sequentiellen Spielen, mit dem Diktatorspiel und Experimente über prosoziales Verhalten, dass erstens ein erheblicher Anteil der Versuchspersonen altruistisch handelt. Zweitens hängt das Ausmaß von verschiedenen ,strukturellen“ Merkmalen der Entscheidungssituation ab. Drittens findet man selbst bei gleicher Struktur der Entscheidungssituation eine ausgeprägte Heterogenität des Verhaltens, wobei die Spannweite von rein eigeninteressiert handelnden Personen bis zu vollständig fairen Aufteilungen reicht (Weimann 1994; Ledyard 1995; Ockenfels 1999; Charness/Rabin 2002; Fehr/Fischbacher 2003; Meier 2006). Dass diese 
Beobachtungen Konsequenzen für die Sozialtheorie und die Erklärung von Kooperation haben, liegt auf der Hand. Insbesondere gilt das für Befunde aus Experimenten über altruistische Reziprozität.

\subsection{Reziprozität}

Die Bedeutung reziproken Handelns für den sozialen Austausch wurde in den klassischen Arbeiten von Soziologie und Ethnologie seit langer Zeit erkannt. Der Traditionsfaden reicht von Simmel (1950) über Malinowski (1926), Mauss (1990), Blau (1964), Homans (1958, 1961), Gouldner (1960) und anderen bis hin zu den modernen Debatten über Sozialkapital (z. B. Fukuyama 2000). Gouldner (1960) hat in seiner Arbeit über die „Norm der Reziprozität“ verschiedene Aspekte reziproken Handelns diskutiert und begriffliche Unterscheidungen eingeführt. Reziprozität erklärt er durch den ,shadow of indebtedness", die intrinsisch verankerte Verpflichtung, positive Leistungen und Zuwendungen zu erwidern. Wir sprechen hier nicht von eigeninteressierter Reziprozität, die in wiederholten Situationen bei genügend großem ,Schatten der Zukunft“ durch spieltheoretische Modelle erklärbar ist (Taylor 1976; Axelrod 1987). Vielmehr geht es um altruistische Reziprozität, z. B. die Erwiderung der Freundlichkeit eines Fremden, also Reziprozität in einmaligen oder endlich oft wiederholten Situationen, in denen das reziproke Handeln nicht der individuell-rationalen Strategie entspricht. „Starke Reziprozität“ („strong reciprocity“) heißt, dass Kooperation mit Kooperation belohnt und Defektion mit Defektion vergolten wird (Gintis 2000; Falk/Fischbacher 2006). Positive und negative altruistische Reziprozität werden in diesem Begriff zusammengeführt (vgl. auch den Überblick zu Experimenten und Konsequenzen für die Sozialtheorie in Fehr/ Gintis 2007). Allerdings definieren wir Reziprozität (anders als z.B. Falk und Fischbacher 2006) nicht durch das Motiv, eine freundlich oder feindlich intendierte Handlung zu erwidern. Die wahrgenommene Intention der Freundlichkeit, aber auch das Motiv der Verringerung von Ungleichheit („Ungleichheitsaversion“) kann reziprokes Handeln erklären. Das Motiv muss aber nicht Bestandteil der Definition von Reziprozität sein. Exprimente zeigen, dass am Zustandekommen altruistischer Reziprozität beide Motive beteiligt sein können (Xiao und Bicchieri 2008).

Anknüpfend an soziologische Hypothesen wendet der Ökonom Akerlof (1982, Akerlof/Yellen 1988) das Prinzip der Reziprozität in der "Gift-Exchange"-Theorie auf die Erklärung von rigiden Löhnen, "Effizienzlöhnen” und Arbeitskosigkeit an.

Löhne und Arbeitseinsatz folgen dem Reziprozitätsprinzip, so dass höhere Löhne mit höherem Arbeitseinsatz vergolten werden. Diese Verbindung von soziologischen Hypothesen und ökonomischen Modellen ist fruchtbar, weil die Theorie dadurch oftmals erweitert und präzisiert wird und $\mathrm{zu}$ neuen, empirisch prüfbaren Hypothesen führt. ${ }^{4}$ Hypothesen der Gift-Exchange-Theorie können im Experiment untersucht werden. Fehr, Fischbacher und Tougarova (2002) berichten über die Befunde eines in Moskau

4 Akerlof ist zudem einer der wenigen Ökonomen, die nicht nur auf soziologischen Befunden aufbauen, sondern diese Tradition auch explizit erwähnen. Der Artikel von Akerlof beginnt mit Studien und einer Beobachtung über Entlohnungsmuster von Homans. Siehe auch Akerlof und Yellen (1988) zur „Fair-Wage-Hypothese“. 
durchgeführten Experiments, bei dem die Auszahlungen an die Versuchspersonen bis zur Höhe eines Monatseinkommens reichten. Das Experiment simuliert einen Arbeitsmarkt, wobei die Probanden die Rolle von Arbeitnehmern und Arbeitgebern einnehmen. Jede von mehreren Spielrunden mit wechselnden Partnern besteht aus zwei Phasen. In der ersten Phase können die Arbeitnehmer in einer Auktion einen Kontrakt mit einer bestimmten Lohnhöhe ersteigern. In der zweiten Phase bestimmen die Arbeitnehmer ihren Arbeitseinsatz. Je höher der Einsatz, desto geringer die Auszahlung an die Arbeitnehmer und desto höher der Gewinn der Arbeitgeber. Hoher Arbeitseinsatz entspricht positiver, altruistischer Reziprozität, falls ein großzügiges Lohnangebot vorlag. Das geringste Niveau entspricht der individuell-rationalen Nash-Gleichgewichtsstrategie. Ebenso können die Arbeitgeber einen hohen Lohn anbieten oder die individuell-rationale Strategie mit dem geringst möglichen Lohn wählen. Wie im Gefangenendilemma ist das NashGleichgewicht nicht pareto-optimal. Arbeitgeber und Arbeitnehmer profitieren von der Kooperation, d. h. von hohen Löhnen und hohem Arbeitseinsatz, aber beide Parteien haben einen Anreiz, sich opportunistisch zu verhalten. Die Prognose der Standardtheorie lautet, dass niedrige Löhne und geringer Arbeitseinsatz gewählt werden. Die Ergebnisse des Experiments widersprechen der ökonomischen Standardtheorie und belegen eindeutig die Prognosen des Gift-Exchange-Modells. Sowohl in der Versuchsbedingung mit relativ geringen Auszahlungen als auch in der Gruppe mit Auszahlungen bis zu einem Monatslohn war bei beiden Parteien, d. h. Spielern in der Rolle von Arbeitnehmern und Arbeitgebern, altruistisch reziprokes Verhalten nachweisbar. Auch in anderen Experimenten und Feldexperimenten ist der Effekt altruistischer, positiver Reziprozität sehr klar ausgeprägt. So im sequentiellen Diktatorspiel, bei dem zunächst Spieler A die Summe auf A und B verteilt und in der zweiten Runde die Rollen wechseln. Selbst bei relativ hohen Auszahlungen wird die Vorleistung in der ersten Runde von Spieler B in der zweiten Runde erwidert (Diekmann 2004). ${ }^{5}$ In der Sozialforschung weiß man, dass die Rücklaufquote bei schriftlichen Befragungen steigt, wenn mit dem Fragebogen ein kleines Geschenk verschickt wird. Ein versprochenes Geschenk erhöht dagegen die Rücklaufquote nicht (Diekmann/Jann 1991). Die Vorleistung des Geschenks aktiviert so zu sagen Gouldners ,shadow of indebtedness“. In der Psychologie demonstriert dies ein Experiment von Regan (1971). Zwei Versuchspersonen befinden sich im Warteraum. Eine Person, tatsächlich ein Mitarbeiter des Versuchsleiters, bietet der Versuchsperson eine Flasche Cola an. Durch diese Aufmerksamkeit steigt die Bereitschaft, angebotene Tombolalose zu kaufen. Gegenüber der Kontrollgruppe (kein Angebot einer Cola) wurden in der Versuchsgruppe fast doppelt so viele Lose gekauft. Nicht-reaktive Daten aus der Beobachtung von Internet-Auktionen liefern ebenfalls eindrucksvolle Belege für reziprokes Handeln. Wenn z. B. der Käufer den Verkäufer mit einer positiven Bewertung nach einer Transaktion belohnt, erhöht sich die Wahrscheinlichkeit, dass der Verkäufer dem Käufer wiederum ein positives Feedback zukommen lässt (Dellarocas et

5 Wobei aber das reziproke Handeln durch Ungleichheitsaversion erklärbar ist. Zwischen dem einfachen Diktatorspiel und dem sequentiellen Spiel mit einer Vorgabe von 50 Prozent unterscheidet sich die Aufteilung von Spieler B nicht signifikant von der Aufteilung im einfachen Diktatorspiel. Die freundliche Intention hat in diesem Experiment keinen Einfluss auf das Ausmaß der Reziprozität. 
al. 2004; Diekmann et al. 2008). ${ }^{6}$ Ohne die Befolgung von Reziprozitätsnormen würden Reputationssysteme bei Internet-Auktionen kollabieren.

Selbstschädigende negative Reziprozität beobachtet man in zahlreichen Experimenten mit dem Ultimatum-Spiel. In diesem Spiel schlägt Person A (der „Proposer") eine Aufteilung vor. Person B (der ,Responder“) kann die Aufteilung akzeptieren oder ablehnen. Lehnt er oder sie ab, gehen beide Spieler leer aus. Gemäß der ökonomischen Standardtheorie sollte B jeden noch so kleinen Betrag akzeptieren, denn etwas ist immer noch besser als nichts. Person A, die eine rationale Entscheidung von B antizipiert, wird nach der Theorie den kleinstmöglichen Betrag anbieten, der von B akzeptiert wird. Spieltheoretisch formuliert ist dies die einzige teilspielperfekte und übrigens auch pareto-optimale Nash-Gleichgewichtsstrategie (von unendlich vielen Gleichgewichtsstrategien), denn jede andere Drohung des Responders (,gib mir mindestens 50 Prozent, sonst lehne ich die Aufteilung ab") wäre keine glaubwürdige Drohung. In Experimenten zeigt sich aber etwas ganz anderes (Gueth et al. 1982; Überblick in Güth/Tietz 1990; Osterbeek et al. 2003). Ein Großteil der Versuchspersonen lehnt zu geringe Angebote ab. Proposer machen Angebote, die im Durchschnitt höher sind als im Diktatorspiel (Forsythe et al. 1994; Ockenfels 1999). Der Grund ist, dass Person A nicht nur ihre Fairnesspräferenzen zum Ausdruck bringt, sondern noch ein zweites Motiv hat. Sie muss die Akzeptanz von B durch ein entsprechend hohes Angebot erreichen, wenn sie nicht leer ausgehen will. Henrich et al. (2000) berichten über Experimente mit Ultimatumspielen bei 15 Naturvölkern wie den Machiguenga in Peru, den Khazax in der Mongolei oder den Aché in Paraguay. Zwar war in allen Kulturen im Gegensatz zur ökonomischen Standardtheorie der Modalwert der Angebote größer als null. Die Varianz der Angebote war aber beträchtlich. Interessanter noch sind die Ablehnungsraten. In einigen Kulturen wurden niedrige Angebote von 20 Prozent und weniger nicht abgelehnt, bei anderen Völkern wie z. B. den Hadza in Tansania oder den Au und Gnau in Papua Neuguinea war es dagegen viel verbreiteter, geringe Angebote abzulehnen. Henrich et al. erklären die Varianz der Proposer-Angebote u. a. durch den Faktor „Marktintegration“. Je mehr eine Kultur an der arbeitsteiligen Marktgesellschaft partizipiert, desto kooperativere Strategien wählen die Teilnehmer an den Experimenten.

Negative und selbst schädigende Reziprozität zeigt sich eindrucksvoll auch in Tierexperimenten mit nahen Verwandten des homo sapiens. In einem Experiment von Brosnan und de Waal (2003) sollten Kapuzineräffchen Nahrung gegen Jetons unter verschiedenen „Fairnessbedingungen“ eintauschen. Gurkenscheiben waren schmackhaft, aber noch begehrter waren Trauben. Gemäß ihrer Präferenzordnung wurde eine Traube einer Gurkenscheibe und diese einem Jeton vorgezogen. In dem Versuch wurde den Kapuzineräffchen eine Gurkenscheibe angeboten, die sie gegen einen Jeton eintauschen sollten. Wenn sie nun mitbekamen, dass Gefährten von ihnen eine Traube für einen Jeton oder sogar kostenlos erhielten, verweigerten sie den Tausch. Es kam sogar vor, dass sie den

6 Dellarocas et al. (2004) erklären die Reziprozität durch strategisches Handeln. Altruistische Motive müssen aber auch eine Rolle spielen, insbesondere wenn Käufer Verkäufer beurteilen, nachdem sie selbst bereits eine Bewertung erhalten haben. Gleiches gilt für Bewertungssysteme, bei denen nur der Verkäufer bewertet wird. Käufer belohnen den guten Ablauf einer Transaktion mit einer positiven Bewertung, wobei das Feedback für den Käufer immerhin mit einem (geringen) Aufwand verbunden ist. 
Versuchsleiter mit der Gurkenscheibe bewarfen. Sie nahmen also Kosten in Kauf, um das ,unfaire“ Verhalten des Versuchsleiters zu sanktionieren.

Gouldners These der Universalität von Reziprozitätsnormen wird durch die experimentelle Wirtschaftsforschung belegt. In Gouldners (1960: 171) Worten: „A norm of reciprocity is, I suspect, no less universal and important an element of culture than the incest taboo, although, similarly its concrete formulations may vary with time and place.“ Eine große Zahl von Studien liefert empirische Evidenz für altruistische, positive Reziprozität und selbst schädigende negative Reziprozität in verschiedensten Kulturen. Es liegt nahe, über Gouldners These noch hinauszugehen und Reziprozitätsnormen als in der Natur des Menschen verankert zu betrachten. Neurowissenschaftliche Untersuchungen mit bildgebenden Verfahren unterstützen diese Vermutung (Rilling et al. 2002).

\subsection{Normen und Sanktionen}

Negative und selbst schädigende Reziprozität wird man im Sinne der oben angegebenen Definition nicht per se als altruistisch bezeichnen. Altruistisch ist diese Form der Reziprozität aber dann, wenn andere Personen von der Revanche profitieren. Dies kann der Fall sein, wenn altruistische Reziprozität die Sanktionierung von Normverletzungen unterstützt. Dass der Vollzug von Sanktionen eine besonders sensible Komponente der faktischen Geltung von Normen darstellt, hat Heinrich Popitz (1961) in seinen Arbeiten über soziale Normen hervorgehoben: „,Der Sanktionen-Vollzug zeigt nicht nur Veränderungen an, er ist selbst der labilste, störungsempfindlichste Teil des normativen Handlungssystems.“ Mit Blick auf die Machtübernahme im nationalsozialistischen Deutschland hat Popitz festgehalten, dass die Schwächung der Normgeltung durch die Schwächung der Sanktionierung von Normbrüchen eingeleitet wird. ,Die Mehrheit der Bevölkerung wird zunächst nicht zum Bruch primärer Normen verleitet, sondern demoralisiert, indem man sie am Vollzug von Sanktionen gegen den Normbruch zu hindern versucht ..." Popitz unterstellt allerdings implizit, dass normalerweise Normbrüche sanktioniert werden. Das ist aber nicht selbstverständlich, wenn Sanktionen nicht nur für die Zielperson, sondern auch für die sanktionierende Person mit Kosten verbunden sind. Wenn die primäre Norm „Kooperation“ vorschreibt, diese Norm verletzt wird und danach eine Sanktionsmöglichkeit besteht, stellt sich die Frage, wer die Sanktionsmöglichkeit ergreifen wird. In der Rational-Choice-Soziologie wird das Sanktionsproblem als „Second-Order-Freerider-Dilemma“ bezeichnet (Heckathorn 1989). Voss (1998, 2001) hat das Sanktionsproblem auf der Basis eines Gefangenendilemmas mit einer zusätzlichen Sanktionsmöglichkeit genauer analysiert. In einem ersten Schritt entscheiden die Akteure simultan über Kooperation oder Defektion. Anschließend werden die Spieler über die Entscheidung ihres Partners informiert. In einem zweiten Schritt haben die Spieler die Möglichkeit, ihren Mitspieler zu sanktionieren. Voss kann nachweisen, dass Kooperation unter bestimmten Bedingungen bezüglich der Auszahlungsstruktur ein Nash-Gleichgewicht darstellt. Dieses Nash-Gleichgewicht ist im einmaligen Spiel aber nur dann teilspielperfekt, wenn keine Sanktionskosten anfallen. Sind Sanktionen mit Kosten verbunden, ist die Sanktionsdrohung ein ,stumpfes Schwert“. Denn der Vollzug der Sanktionsdrohung ist nicht mehr glaubwürdig, was rationale Akteure antizipieren. 
Sie werden defektieren, weil sie nicht mit einer Sanktion rechnen müssen, so dass keine Kooperation entsteht und die Norm nicht aufrechterhalten werden kann. Erweitert man nun das Standardmodell der Rationalitätstheorie durch Annahmen über altruistische Reziprozität, so kann das Sanktionsdilemma gelöst werden. Wenn Personen selbst schädigende Sanktionen ergreifen, ist Kooperation unter bestimmten Bedingungen (bezüglich der Auszahlungsstruktur im sozialen Dilemma, der Sanktionskosten und der Sanktionshöhe) eine teilspielperfekte Nash-Gleichgewichtsstrategie (Fehr/Schmidt 1999, zum Gefangenendilemma-Normspiel mit Sanktionen s. Diekmann/Voss 2008).

Soweit die Theorie. Wie aber verhalten sich Akteure tatsächlich in einem sozialen Dilemma mit altruistischen Sanktionsmöglichkeiten? Fehr und Gächter (2002) haben ein Public-Good-Game (PG) durch die zusätzliche Möglichkeit zur Sanktionierung erweitert (PGS). Das Spiel wurde mit vier Personen pro Gruppe in jeweils unterschiedlicher Gruppenzusammensetzung über 12 Runden gespielt. In den ersten sechs Runden wurde das PGS, in den anschließenden sechs Runden das PG ohne Sanktionsmöglichkeit gespielt (in einer zweiten Versuchsbedingung wurde die Reihenfolge vertauscht). Die Teilnehmer erhalten für jede Spielrunde 20 Geldeinheiten (GE). Diese können sie ganz oder teilweise in einen Fonds investieren. Der Fonds hat eine Wachstumsrate von 60 Prozent und wird gleich unter alle vier Akteure aufgeteilt. Investieren alle Akteure im Fall vollständiger Kooperation in den Fonds, so erzielen sie eine Auszahlung von 32 GE $(=80 \cdot 1,6 / 4)$. Jede Person hat aber einen Anreiz, von der kooperativen Strategie abzuweichen. Im Nash-Gleichgewicht folgen alle Spieler der individuell-rationalen Strategie und erhalten nur je 20 Punkte. Im PGS werden die Spieler nach der Investition über die Entscheidungen der Mitspieler informiert. Sie können sich sodann für eine abgestufte Sanktionierung von Mitspielern entscheiden. Jede Sanktionierung eines Mitspielers kostet zwischen einem und $10 \mathrm{GE}$, wobei der sanktionierte Spieler eine drei Mal so hohe Einbuße erleidet. Das Ergebnis des Experiments zeigt, dass die 240 Versuchspersonen im Gegensatz zur ökonomischen Standardtheorie häufig von der Möglichkeit selbst schädigender Bestrafung Gebrauch machten. 84 Prozent der Versuchspersonen übten mindestens einmal eine Sanktion aus. Je geringer das Kooperationsniveau einer Person im Vergleich zum Gruppendurchschnitt war, desto härter fiel die Bestrafung aus. Die Sanktionierung führt zu einer stark erhöhten Bereitschaft zur Investition in den Fonds. In der Sanktionierungsphase haben 94 Prozent der Versuchspersonen im Durchschnitt mehr investiert als im einfachen PG ohne Sanktionsmöglichkeit; bei umgekehrter Reihenfolge der beiden Spielvarianten waren es 91 Prozent. Die Möglichkeit zu altruistischer Sanktionierung bewirkt eine drastische Erhöhung des Kooperationsniveaus. Dieses Experiment wurde kürzlich weltweit in 16 Städten wie Kopenhagen, Boston, Seoul, Minsk, Samara, Istanbul, Muscat, Riad u. a. durchgeführt (Herrmann et al. 2008). Eindeutig zeigte sich in allen Experimenten, dass Trittbrettfahrer altruistisch sanktioniert wurden. In vielen Gesellschaften wurden aber nicht nur Trittbrettfahrer, sondern auch Mitspieler bestraft, die sich durch ein überdurchschnittlich hohes Kooperationsniveau ausgewiesen hatten. Erklärbar ist „,antisocial punishment“ durch Reziprozität. Bestrafte Trittbrettfahrer neigen dazu, an überdurchschnittlich kooperativen Mitspielern Vergeltung zu üben. Die Tendenz zur Sanktionierung prosozialen Verhaltens variiert mit der Rechtskultur einer Gesellschaft. Sind rechtliche Institutionen zur Regelung von Konflikten unter Fremden schwach verankert und zivile Normen 
kooperativen Verhaltens nur in geringem Maße ausgeprägt, ist die Sanktionierung der überdurchschnittlich Kooperativen besonders häufig zu beobachten. Durch die Sanktionierung prosozialen Verhaltens geht das Kooperationsniveau wieder zurück, in einigen Experimenten sogar unter das Niveau der Kontrollgruppe ohne Sanktionsmöglichkeit. Bemerkenswert an den komparativen Experimenten ist die Erklärung kultureller Unterschiede. Rechtliche Institutionen und Normen ziviler Kooperation spielen dabei eine Schlüsselrolle. $\mathrm{Ob}$ die gefundenen Korrelationen kausal gedeutet werden können, ist wie in vielen anderen makrosoziologischen Studien aber spekulativ und bedarf zur Absicherung detaillierter Untersuchungen.

Was passiert, wenn in einer Gesellschaft eine Art Wettbewerb von Institutionen herrscht und Personen frei wählen können, ob sie sich an einem PG oder einem PGS beteiligen? Man könnte sich z. B. vorstellen, Studenten wählen eine Wohngemeinschaft aus und entscheiden darüber, ob sie lieber in einer Gemeinschaft vom Typ PG oder in einer Gemeinschaft mit Sanktionsinstitution vom Typ PGS leben möchten. Gürerk, Irlenbusch und Rockenbach (2006) haben einen solchen Wettbewerb im Experiment untersucht. Anfangs wählten die meisten Versuchspersonen das PG. Wegen des höheren Kooperationsniveaus im PGS wechselten im Laufe der Zeit mehr und mehr Personen die Spielsituation. Nach 30 Spielrunden befanden sich mehr als 90 Prozent der Versuchspersonen in der PGS-Situation, in der die Teilnehmer fast vollständig kooperierten, während die im PG verbleibende Minderheit vollständig defektierte.

Die Experimente demonstrieren den hohen Stellenwert negativer und altruistischer Reziprozität für die Befolgung sozialer Normen und die Aufrechterhaltung von Kooperation. Man kann mit Recht sagen, dass Reziprozität der Kitt ist, der die Gesellschaft insbesondere dort zusammenhält, wo einklagbare Rechtsnormen keine Wirkung entfalten oder nur einen Rahmen definieren. Die Experimente der Wirtschaftsforschung und anderer Disziplinen tragen mit ihren Befunden wesentlich zur Weiterentwicklung der Sozialtheorie bei.

\section{Auf der Suche nach dem erklärenden Modell}

Viele Einzelergebnisse widersprechen der ökonomischen Standardtheorie. Damit fehlt aber auch der theoretische Rahmen für die zahlreichen Befunde. Gibt es alternative Modelle, die das Puzzle der Effekte aus den Experimenten mit Varianten von Diktator-, Ultimatum-, Public-Good- und anderen Kooperationsspielen erklären können? Neben lerntheoretischen Ansätzen (Erev/Roth 1998) wurden verschiedene Fairness-Theorien vorgeschlagen. Das Modell von Rabin (1993) setzt bei den wahrgenommenen Intentionen des Mitspielers an und definiert auf dieser Basis ein so genanntes Fairness-Gleichgewicht. Eine andere und einfachere Möglichkeit, Theorie und experimentelle Ergebnisse zu versöhnen ist die Idee der Ungleichheitsaversion. Kern der Modelle ist eine Nutzenfunktion, die neben den materiellen Auszahlungen als zusätzliches Argument Fairnesspräferenzen berücksichtigt. Die Modelle unterscheiden sich durch die Wahl der Funktion für die Ungleichheitsaversion. Im ERC-Modell von Bolton und Ockenfels (2000, Ockenfels 1999) - ERC steht für „Equity, Reciprocity und Competition“ - wird die Klasse zulässiger Funktionen durch eine Reihe von Axiomen festgelegt. Al- 
len Funktionen gemeinsam ist die folgende Eigenschaft: Der Nutzenverlust durch Ungleichheitsaversion ist umso größer, je größer der Anteil eines Spielers an der Summe aller Auszahlungen vom Referenzpunkt der durchschnittlichen Auszahlung abweicht. Die Fairnesstheorie von Fehr und Schmidt (1999) hebt nicht auf den Vergleich der relativen Auszahlung mit dem Referenzpunkt ab, sondern vergleicht die Auszahlung an Person i mit den Auszahlungen an alle anderen Mitspieler. Höhere Auszahlungen (Unfairness) können dabei anders gewichtet werden als geringere Auszahlungen (die Neidkomponente). Das Modell ist relativ einfach, so dass die Grundidee gut an diesem Modell demonstriert werden kann. Wir beschränken uns auf den Zwei-Personen-Fall:

$$
\mathrm{u}_{\mathrm{i}}\left(\mathrm{x}_{\mathrm{i}}\right)=\mathrm{x}_{\mathrm{i}}-\alpha_{\mathrm{i}} \max \left\{\mathrm{x}_{\mathrm{j}}-\mathrm{x}_{\mathrm{i}} ; 0\right\}-\beta_{\mathrm{i}} \max \left\{\mathrm{x}_{\mathrm{i}}-\mathrm{x}_{\mathrm{j}} ; 0\right\} .
$$

$\mathrm{u}_{\mathrm{i}}$ ist der Nutzen der Auszahlung $\mathrm{x}_{\mathrm{i}}$ an Akteur i. $\alpha_{\mathrm{i}}$ und $\beta_{\mathrm{i}}$ sind Personenparameter, wobei gilt: $0 \leq \beta_{\mathrm{i}}<1 ; \beta_{\mathrm{i}} \leq \alpha_{\mathrm{i}}$. Der erste Term auf der rechten Seite bezeichnet die materiellen Auszahlungen, der zweite Term bezieht sich auf die Neidkomponente einer Auszahlung und der dritte Term auf die Unfairness der Auszahlung. $\alpha \geq \beta$ heißt, dass der immaterielle Nutzenverlust durch Unfairness gleich stark oder stärker gewichtet wird als der Nutzenverlust durch Privilegierung (Fehr/Schmidt 1999: 822).

In einer Entscheidungssituation besteht oftmals ein Zielkonflikt zwischen materiellen Auszahlungen und Fairnessüberlegungen. Höhere Auszahlungen steigern den Nutzen über den materiellen Term, können aber gleichzeitig den Nutzen des Fairnessterms verringern.

Das Modell formalisiert diesen Zielkonflikt. Betrachten wir als Beispiel das Gefangenendilemma mit den Auszahlungen T $>\mathrm{R}>\mathrm{P}>\mathrm{S}$. Der mit dem Fairnessmodell transformierte Nutzen eines Ausbeutungsgewinns $\mathrm{T}$ und eines Kooperationsgewinns $\mathrm{R}$ beträgt unter Verwendung der oben erwähnten Nutzenfunktion: $u(T)=T-\beta(T-S)$ und $u(R)=$ $R$. Falls $u(R) \geq u(T)$ existiert im Gefangenendilemma ein kooperatives Nash-Gleichgewicht von Fairnessauszahlungen. Die Bedingung dafür lautet: $\beta>(T-R) /(T-S)$. Sind die Fairnessparameter beider Akteure im Gefangenendilemma hinreichend groß, werden sie ein kooperatives Nash-Gleichgewicht erreichen.

Die Personenparameter $\alpha$ und $\beta$ können durch die empirisch beobachteten Entscheidungen ermittelt werden. Ohne Messung von $\alpha$ und $\beta$ erlaubt das Modell eine Vielzahl von qualitativen Vorhersagen, die mit Ergebnissen aus Experimenten mit dem Ultimatumspiel, Public-Good-Games und vielen anderen (aber keineswegs allen) Entscheidungssituationen im Einklang stehen. Eine Schwäche der einfachen linearen Funktion fällt im Diktatorspiel auf. Für $\beta<0,5$ prognostiziert die Theorie eine vollständig eigeninteressierte Aufteilung und für $\beta>0,5$ eine vollständig faire 50/50 Verteilung. Erst wenn man die Linearitätsannahme aufgibt, stimmen die Prognosen mit der beobachteten Verteilung überein (Fehr/Schmidt 1999). Aber auch die Konkurrenzmodelle haben ihre schwachen Punkte. Engelmann und Strobel (2004) haben das ERC-Modell und das Fehr-Schmidt-Modell in Testsituationen verglichen, in denen die beiden Modelle unterschiedliche Aufteilungen prognostizieren. Bei diesem Test hat das Fehr-SchmidtModell besser als das ERC-Modell abgeschnitten, aber natürlich hängt die Trefferquote auch von den ausgewählten Testsituationen ab. Beide Modelle kennen neben der Präferenz für materielle Auszahlungen das zusätzliche Motiv der Ungleichheitsaversion. 
Unter bestimmten Bedingungen kann aber auch das gegenteilige Motiv auftreten. Im Falle von Statuswettbewerb steigt der Nutzen mit dem Abstand von der Auszahlung an den Mitspieler (Bolton/Ockenfels 2000). Dieser gegenläufige Effekt wird in beiden Modellen ausgespart. Tutic/Liebe (2008) haben das Fehr-Schmidt-Modell durch den Einbezug von Status erweitert und verschiedene, prüfbare Modellkonsequenzen abgeleitet. In Experimenten ist zudem beobachtbar, dass neben materiellen Auszahlungen, Ungleichheitsaversion und Wettbewerb weitere Motive eine Rolle spielen. Charness und Rabin (2002) weisen mit einer Serie von Diktatorspielen nach, dass bei Heterogenität der Motive die Entscheidungen von mehr Personen durch Wohlfahrtsmaximierung (Pareto-Effizienz) als durch Ungleichheitsaversion erklärbar sind. Kooperatives Verhalten im einmaligen Gefangenendilemma z. B. ist sowohl durch Ungleichheitsaversion als auch mit dem Motiv der Wohlfahrtsmaximierung erklärbar. Um zwischen beiden Hypothesen diskriminieren zu können, muss man ein „experimentum crucis“ arrangieren. Mit dem einfachen, binären Diktatorspiel sieht das folgendermaßen aus: Diktator B kann zwischen den Aufteilungen $(400,400)$ und $(750,400)$ wählen, wobei jeweils der erste Wert in der Klammer an A und der zweite Wert an B ausgezahlt wird. Die meisten Versuchspersonen (69 Prozent) wählen, im Gegensatz zur Hypothese der Ungleichheitsaversion, die effiziente, aber ungleiche Aufteilung, die A materiell begünstigt, ohne dass die materielle Auszahlung an B geschmälert wird. Selbst wenn die Unterstützung von A etwas kostet und die Ungleichheit wächst, wählt die Hälfte der Versuchspersonen bei der Entscheidung zwischen den Verteilungen $(400,400)$ und $(750,375)$ immer noch die wohlfahrtsmaximierende Aufteilung.

Die Theorien von Fehr und Schmidt und Bolton und Ockenfels modifizieren die Nutzenfunktion, indem neben den materiellen Auszahlungen Ungleichheitsaversion als weitere Nutzenkomponente berücksichtigt wird. Man könnte nun kritisch einwenden, dass einfach an der „Präferenzschraube“ gedreht wird, um Theorie und Beobachtungen durch Ad-hoc-Modifikationen in Übereinstimmung zu bringen. Dieser Einwand ist aber nicht gerechtfertigt, denn Motive werden nicht direkt „gemessen“ (wie etwa bei manchen Anwendungen der SEU-Theorie oder der Ajzen-Fishbein-Theorie in Soziologie und Sozialpsychologie), sondern indirekt über die Auszahlungsrelationen von Spieler und Mitspieler erfasst. Argumente der Nutzenfunktion sind ausschließlich materielle Auszahlungen, so dass sich auch keine Skalen- oder Messprobleme ergeben. Die Modelle sind, auch im wissenschaftstheoretischen Sinne, nicht ad-hoc, weil sie die Möglichkeit bieten, eine Vielzahl neuer, potentiell falsifizierbarer (und teilweise auch bereits falsifizierter) Konsequenzen abzuleiten, wobei sie auf die elaborierten formalen Hilfsmittel der Spieltheorie zurückgreifen können. Positive und negative Reziprozität z. B. ist in den Theorien der Ungleichheitsaversion kein eigenständiges Motiv, sondern eine Folge von Ungleichheitsaversion. Andere Motive wie Statuswettbewerb (Tutic/Liebe 2008) und Wohlfahrtsmaximierung (Charness/Rabin 2002) können in ähnlicher Weise durch eine geeignete Verknüpfung mit den Auszahlungsrelationen berücksichtigt werden.

Diese Modelle haben eine Eigenschaft gemeinsam. Sie prognostizieren, dass die Entscheidungen von Personen nur vom „outcome“, von der Verteilung der Auszahlungen auf die Akteure abhängen. Intentionen von Mitspielern, freundliche oder feindselige Akte in vorhergehenden Entscheidungen sequentieller Spiele haben nur einen Einfluss über die letztlich erzielte Verteilung. Betrachten wir dagegen folgendes Experiment mit 
dem Vertrauensspiel (McCabe et al. 2003; Vieth/Weesie 2007). In der Variante (1) kann der Treugeber vertrauen oder defektieren. Wählt er letztere Option, erhalten beide Akteure die Auszahlung $(20,20)$ und das Spiel ist beendet. Vertraut der Treugeber, geht die Entscheidung an den Treuhänder. Der Treuhänder hat die Möglichkeit zu kooperieren oder zu defektieren. Im Falle der Kooperation ist die Auszahlung (25, 25), bei Defektion $(15,30)$, wobei 15 Punkte an den Treugeber und 30 Punkte auf den Treuhänder entfallen. Bei Variante (2) kann der Treuhänder direkt und ohne Vertrauensvorgabe zwischen den Alternativen Kooperation und Defektion wählen. Obwohl bei beiden Varianten der Treuhänder mit der gleichen Entscheidung zwischen Kooperation und Defektion mit den Auszahlungen $(25,25)$ versus $(15,30)$ konfrontiert ist, entscheidet er sich häufiger für Kooperation, wenn der Treugeber einen Vertrauensvorschuss gegeben hat (65 Prozent versus 33 Prozent im Experiment von McCain et al.) Im Vertrauensspiel „eingebettet“ ist ein Diktatorspiel (Vieth/Weesie 2007, wobei hier die im Experiment beobachtete Differenz aber wesentlich geringer ist). Vergleicht man das eingebettete mit dem isolierten Diktatorspiel und ergeben sich Unterschiede, können verteilungsorientierte Modelle diese Varianz nicht erklären. Das einfache Experiment demonstriert den Einfluss der Intention; ein Vertrauensvorschuss wird honoriert. Die Bestrafung unfreundlicher und die Belohnung freundlicher Handlungen in sequentiellen Spielen wird von Yang, Weimann und Mitropoulos (2006) nicht mit Reziprozitätsmotiven oder durch Ungleichheitsaversion, sondern alternativ mit einer „Stick-and-Carrot-Heuristik“ erklärt, wobei dem Belohnungs- und Bestrafungspotential (der Verhandlungsmacht) eine Schlüsselrolle zukommt. Charness und Rabin (2002) entwickeln ein Modell, das Ungleichheitsaversion, Wohlfahrtsmaximierung und die wahrgenommene Intention simultan berücksichtigt, allerdings um den Preis der Einführung weiterer Parameter. Neben Modellen, die die Nutzenfunktion modifizieren, die Standard-Rationalitätstheorie aber beibehalten, gehen Theorien begrenzter Rationalität andere Wege, indem sie z. B. das Verhalten in Entscheidungssituationen durch einfache heuristische Prinzipien erklären (Gigerenzer/Todd 1999).

Der Wettbewerb von Theorien und Modellen strukturiert die Forschung und gibt Richtungen vor, in die sich die experimentelle Forschung bewegt. Die vorliegenden Modelle sind ein produktiver Beginn, aber sicher noch nicht das letzte Wort bei dem Versuch, die mannigfaltigen experimentellen Ergebnisse theoretisch zu integrieren.

\section{Ausblick}

Die skizzierten Theorien und Hypothesen über soziale Interaktionen in Konfliktsituationen und sozialen Dilemmata, über die Entstehung und Stabilisierung sozialer Normen und sozialer Ordnung, über Altruismus, Reziprozität und andere Kernprobleme der Sozialtheorie bilden die Grundlage eines interdisziplinären und kumulativen Forschungsprogramms. Dieses Programm spannt den Bogen für die Weiterentwicklung von Theorien und empirische Prüfungen. Weiter oben wurde der methodische Status von Experimenten als „Wahrheitsfilter“ beschrieben. Das ist aber nur bedingt richtig, denn es gibt bekanntlich $\alpha$ - und $\beta$-Fehler. Mit Blick auf die Biomedizin hat Ioannidis (2005) in einem provokanten Artikel darauf aufmerksam gemacht, dass sich selbst bei regel- 
gerechter und korrekter statistischer Analyse (was keineswegs immer der Fall ist) statistisch-signifikante, aber fehlerhafte Ergebnisse in der veröffentlichten Literatur häufen. ${ }^{7}$ Mit Replikationen erhöht sich die Wahrscheinlichkeit drastisch, dass fehlerhafte Ergebnisse aussortiert werden. Ein statistisch-signifikanter Effekt, erstmalig in der Literatur berichtet, ist nicht viel mehr als eine Hypothese. Signifikante Effekte sollte man erst dann zum Nennwert nehmen, wenn sie in Folgestudien reproduzierbar sind. Deshalb sind methodisch sorgfältige Replikationen für ein kumulatives, empirisch orientiertes Forschungsprogramm so bedeutsam (vgl. auch Villeval 2007).

In dem vorliegenden Artikel haben wir uns auf die drei zentralen und aufeinander aufbauenden Themenkreise von Altruismus, altruistischer Reziprozität und sozialen Normen beschränkt. Ein weiterer Schwerpunkt der experimentellen Wirtschaftsforschung ist das Vertrauensproblem. In diesem Themenbereich war zunächst die Soziologie federführend (Gambetta 1988; Coleman 1990; Raub 1992; Preisendörfer 1995; Snijders 1996) und auch heute noch ist die soziologische Forschung mit experimentellen und anderen Methoden an der Forschung über Vertrauensprobleme stark beteiligt (Raub/Buskens 2007). ${ }^{8}$

Die soziologische Forschung hat zudem eine starke Tradition in der Analyse sozialer Netzwerke. Durch die Verknüpfung von sozialen Netzwerken mit dem Vertrauens- und Kooperationsproblem (Raub/Weesie 1990) oder mit sozialem Tausch in der NetzwerkAustauschtheorie (s. z. B. Braun/Gautschi 2006), durch die Berücksichtigung also von Formen „sozialer Einbettung“, wurde die zumindest damals verkürzte ökonomische Optik wesentlich erweitert. Heute werden klassische soziologische Hypothesen über Reziprozität, soziale Normen und Sanktionen in der experimentellen Wirtschaftsforschung mit Experimenten und Feldexperimenten untersucht. Mit den Modellen von Ökonomie, Spieltheorie und „Behavioral Game Theory“ werden neue Hypothesen formuliert, klassische Hypothesen präzisiert, empirisch geprüft und eventuell modifiziert. Diese Verbindung von Soziologie, Ökonomie und experimentellen Studien gibt der Entwicklung der Sozialtheorie neue Impulse, die von der soziologischen Theorie nicht länger ignoriert werden können. Die Ökonomie sollte auf der anderen Seite den interdisziplinären Charakter des Forschungsprogramms stärker herausstreichen. Neben Ökonomie

7 Je nach Annahmen (zentral ist dabei die Apriori-Wahrscheinlichkeit eines Effekts in einem Untersuchungsbereich) kann man anhand von Rechenbeispielen, mit Simulationen und der Formel von Bayes ermitteln, dass unter durchaus realistischen Annahmen ein Großteil der als signifikant publizierten Ergebnisse falsch ist.

8 Experimentelle und andere empirisch-soziologische Forschungen zum Vertrauensspiel, glaubwürdigen Verpflichtungen („commitments“, Weesie/Raub 1996) zur Lösung des Vertrauensproblems und Anwendungen im Bereich der Wirtschaftssoziologie werden in dem Überblick von Raub und Buskens (2007) behandelt. Eine große Rolle spielen auch Koordinationsprobleme. Anwendungen finden sich besonders im Bereich des Verkehrsverhaltens. Zu einer experimentellen Untersuchung von Koordinationsproblemen beim Routenwahlspiel siehe Helbing et al. (2005). Eine interessante Anwendung von Nullsummenspielen auf die Interaktion von Torwart und Elfmeterschützen findet man in Berger/Hammer 2007. Schließlich sei noch auf eine Forschungsrichtung hingewiesen, die für die Soziologie größere Bedeutung haben könnte. Die Untersuchung insbesondere kostenintensiver Konmmunikation mittels Signalspielen (zu Anwendungen Posner 2000, Gambetta/Hamill 2005, Diekmann/Przepiorka 2007. Überblick Bliege Bird/Smith 2005). 
und Soziologie sind Forscherinnen und Forscher aus Politikwissenschaft, Sozialpsychologie, Ethnologie, Biologie und Rechtswissenschaft (Posner 2000; McAdams 2000) an diesem Programm beteiligt. Der Terminus „experimentelle Wirtschaftsforschung“ wird mehr und mehr zu einem irreführenden Begriff.

Die empirische Forschung kann, mit Blick auf die Methoden, von den Forschungen lernen, dass zur Methodenvielfalt auch Experimente und Feldexperimente gehören. „Ist Soziologie eine nomographische Wissenschaft, und erhebt sie den Anspruch, Regeln aufzustellen, die in exakter Weise die funktionalen und kausalen Zusammenhänge auf dem Gebiete der sozialen Erscheinungen beschreiben, so muss früher oder später der Zeitpunkt kommen, an dem ihre Probleme experimentell untersucht werden." In dem Aufsatz in der „Kölner Zeitschrift“ aus dem Jahre 1928, aus dem dieses Zitat stammt, berichtet Pitirim A. Sorokin die Ergebnisse eines Experiments über „Arbeitsleistung und Entlohnung“. Sein Plädoyer für Experimente in der Soziologie hat heute an Aktualität nicht verloren.

\section{Literatur}

Albert, Hans, 1965. Modell-Platonismus. Der neoklassische Stil des ökonomischen Denkens in kritischer Beleuchtung, in: Topitsch, Ernst (Hrsg.), Logik der Sozialwissenschaften. Köln: Kiepenheuer.

Albert, Hans, 2008. Bemerkungen zum Problem der Erklärung sozialer Vorgänge. Max Webers Synthese und die modernen Sozialwissenschaften, in: Diekmann, Andreas/Eichner, Klaus/ Schmidt, Peter/Voss, Thomas (Hrsg.), Rational Choice: Theoretische Analyse und empirische Resultate. Festschrift für Karl-Dieter Opp. Wiesbaden: VS-Verlag.

Akerlof, George A., 1982: Labor Contracts as Partial Gift Exchange, in: The Quarterly Journal of Economics 47, 543-569.

Akerlof, George A., Janet L., 1988: Fairness and Unemployment, in: American Economic Review 78, 44-49.

Andreoni, James/Miller, Joh, 2002: Giving According to GARP: An Experimental Test of the Consistency of Preferences for Altruism, in: Econometrica 70, 737-753.

Axelrod, Robert, 1987 [1984]: Die Evolution der Kooperation. München: Oldenbourg.

Becker, Gary S., 1981: A Treatise on the Family. Cambridge, Mass: Harvard University Press.

Benenson, Joyce F./Pascoe, Joanna/Radmore, Nicola, 2007. Children's Altruistic Behavior in the Dictator Game, in: Evolution and Human Behavior 28, 168-175.

Benz, Matthias/Meier, Stephan, 2006: Do People Behave in Experiments as in the Field? Evidence From Donations. Working paper No. 06-8 der Federal Reserve Bank of Boston, Research Center for Behavioral Economics and Decision Making.

Berger, Roger/Hammer, Rupert, 2007: Die doppelte Kontingenz von Elfmeterschüssen, in: Soziale Welt 58, 397-418.

Blau, Peter M., 1964: Exchange and Power in Social Life. New York: Wiley.

Bliege Bird, Rebecca/Smith, Eric A., 2005: Signaling Theory, Strategic Interaction, and Symbolic Capital, in: Current Anthropology 46, 221-248.

Bolton, Gary E./Ockenfels, Axel, 2000: ERC: A Theory of Equity, Reciprocity, and Competition, in: American Economic Review 90, 166-93.

Braun, Norman/Gautschi, Thomas, 2006: A Nash-Bargaining Model for Simple Exchange Networks, in: Social Networks 28, 1-23.

Brosnan, Sarah F./de Waal, Frans B. M., 2003: Monkeys Reject Unequal Pay, in: Nature 425, 297-299. 
Bungart, W./Lück, H. E., 1974. Forschungsartefakte und nicht-reaktive Meßverfahren, Stuttgart: Teubner.

Camerer, Colin F., 1997: Progress in Behavioral Game Theory, in: Journal of Economic Perspectives 11, 167-188.

Camerer, Colin F., 2003: Behavioral Game Theory. Experiments in Strategic Interaction. Princeton, New Jersey: Princeton University Press.

Charness, Gary/Rabin, Matthew, 2002: Understanding Social Preferences with Simple Tests, in: The Quarterly Journal of Economics 117, 817-869.

Charness, Gary/Rabin, Matthew, 2002: Understanding Social Preferences with Simple Tests, in: The Quarterly Journal of Economics 117, 817-869.

Cherry, Todd L./Frykblom, Peter/Shogren, Jason F., 2002: Hardnose the Dictator, in: American Economic Review 92, 1218-1221.

Coleman, James S., 1990: Foundations of Social Theory. Cambridge, Mass.: Harvard University Press.

Darley, J. M./Latané, B., 1968: Bystander Intervention in Emergencies. Diffusion of Responsibility, in: Journal of Personality and Social Psychology 8, 377-383.

Deci, Edward L., 1975: Intrinsic Motivation. New York: Plenum Press.

Dellarocas, Chrysanthos/Fan, Ming/Wood, Charles A., 2004: Self-Interest, Reciprocity, and Participation in Online Reputation Systems. MIT Center for Digital Business, Working Paper 205.

Diekmann, Andreas, 1985: Volunteer's Dilemma, in: Journal of Conflict Resolution 29, 605-610.

Diekmann, Andreas, 1993: Cooperation in an Asymmetric Volunteer's Dilemma Game, in: International Journal of Game Theory 22, 75-85.

Diekmann, Andreas, 2004: The Power of Reciprocity. Fairness, Reciprocity, and Stakes in Variants of the Dictator Game, in: Journal of Conflict Resolution 48, 487-505.

Diekmann, Andreas/Jann, Ben, 2001: Anreizformen und Ausschöpfungsquoten bei postalischen Befragungen. Eine Prüfung der Reziprozitätshypothese, in: ZUMA-Nachrichten 48, 18-26.

Diekmann, Andreas/Voss, Thomas, 2008: Soziale Normen und Reziprozität. Die Bedeutung ,sozialer" Motive für die Rational-Choice-Erklärung sozialer Normen, in: Diekmann, Andreas/ Eichner, Klaus/Schmidt, Peter/Voss, Thomas (Hrsg.), Rational Choice: Theoretische Analyse und empirische Resultate. Festschrift für Karl-Dieter Opp. Wiesbaden: VS-Verlag.

Diekmann, Andreas/Przepiorka, Wojtek, 2007: Signaling Trustworthiness. Evidence from Lab Experiments, Mimeo: ETH Zürich.

Diekmann, Andreas/Jann, Ben/Przepiorka, Wojtek/Wehrli, Stefan, 2008. The Evolution of Cooperation on Anonymous Markets. Mimeo: ETH-Zürich.

Engelmann, Dirk/Strobel, Martin, 2004: Inequality Aversion, Efficiency, and Maximin Preferences in Simple Distribution Experiments, in: American Economic Review 94, 857-869.

Erev, Ido/Roth, Alvin E., 1998. Predicting How People Play Games: Reinforcement Learning in Experimental Games with Unique, Mixed Strategy Equilibria, in: The American Economic Review 88: 848-881.

Falk, Arnim/Fischbacher, Urs, 2006: A Theory of Reciprocity, in: Games and Economic Behavior 54, 293-315.

Fischbacher, Urs, 2006: z-Tree: Zurich Toolbox for Ready-Made Economic Experiments. Institut für empirische Wirtschaftsforschung der Universität Zürich: Mimeo.

Fehr, Ernst/Fischbacher, Urs/Tougarova, Elena, 2002: Do High Stakes and Competition Undermine Fairness? Evidence from Russia. Working paper No. 120. Institute for Empirical Research in Economics, University of Zurich.

Fehr, Ernst/Fischbacher, Urs, 2003: The Nature of Human Altruism, in: Nature 425, 785-791. Fehr, Ernst/Gächter, Simon, 2002: Altruistic Punishment in Humans, in: Nature 415, 137-140. Fehr, Ernst/Herbert, Gintis, 2007: Human Motivation and Social Cooperation: Experimental and Analytical Foundations, in: Annual Review of Sociology 33, 43-64. 
Fehr, Ernst/Schmidt, Klaus M., 1999: A Theory of Fairness, Competition, and Cooperation, in: The Quarterly Journal of Economics 114, 817-868.

Frey, Bruno S./Jegen, Reto, 2001: Motivation Crowding Theory. A Survey of Empirical Evidence, in: Journal of Economic Surveys 5, 589-611.

Frey, Bruno S., 1997. Not Just for the Money. An Economic Theory of Personal Motivation. Brookfield: Edward Elgar.

Friedrichs, Jürgen (Hrsg.), 1973. Teilnehmende Beobachtung abweichenden Verhaltens. Stuttgart: Enke.

Forsythe, Robert/Horowitz, Joel L./Savin, N. E./Sefton, Martin, 1994: Fairness in Simple Bargaining Experiments, in: Games and Economic Behavior 6, 347-369.

Fukuyama, Francis, 2000: Social Capital and Civil Society. International Monetary Fund: Working paper $00 / 74$.

Gambetta, Diego (Hrsg.), 1988: Trust. Making and Breaking Social Relations. Oxford: Basil Blackwell.

Gambetta, Diego/Hamill, Heather, 2005: Streetwise. How Taxidriver Establish their Customers' Trustworthiness. New York: Russell Sage Foundation.

Gigerenzer, Gerd, 2000: Adaptive Thinking. Rationality in the Real World. Oxford: Oxford University Press.

Gigerenzer, Gerd/Todd, Peter M., 1999: Simple Heuristics that Make us Smart. Oxford: Oxford University Press.

Gneezy, Uri/Rustichini, Aldo, 2000a. A Fine is a Price, in: The Journal of Legal Studies 29, 1-17.

Gneezy, Uri/Rustichini, Aldo, 2000b. Pay Enough or Don't Pay at All, in: The Quarterly Journal of Economics 115, 791-810.

Goette, Lorenz/Stutzer, Alois, 2008. Blood Donations and Incentives. Evidence from a Field Experiment Research Center for Behavioral Economics and Decision Making. Federal Reserve Bank Boston. Arbeitspapier 08-3.

Gouldner, Alvin W., 1960: The Norm of Reciprocity: A Preliminary Statement, American Sociological Review 25, 161-178.

Gueth, Werner/Schmittberger, Rolf/Schwarze, Bernd, 1982: An Experimental Analysis of Ultimatum Bargaining, in: Journal of Economic Behavior and Organization 3, 367-388.

Güth, Werner/Tietz, Reinhard, 1990: Ultimatum Bargainig Behavior. A Survey and Comparison of Experimental Results, in: Journal of Economic Psychology 11, 417-449.

Gürerk, Özgür/Irlenbusch, Bernd/Rockenbach, Bettina, 2006: The Competetive Advantage of Sanctioning Institutions, in: Science 312, 108-111.

Häring, Norbert/Storbeck, Olaf, 2007: Ökonomie 2.0. 99 überraschende Erkenntnisse. Stuttgart: Schaeffer-Poeschel.

Harrison, Glenn W./List, John A., 2004: Field Experiments, in: Journal of Economic Literature 42, 1009-1055.

Heckathorn, Douglas D., 1989: Collective Action and the Second Order Free-Rider Problem, in: Rationality and Society 1, 78-100.

Helbing, Dirk/Schönhof, Martin/Stark, Hans-Ulrich/Holyst, Janusz A., 2005. How Individuals Learn to Take Turns: Emergence of Alternating Cooperation in a Congestion Game and the Prisoner's Dilemma, in: Advances in Complex Systems 8, 87-116.

Henrich, Joseph/Boyd, Robert/Bowles, Samuel/Camerer, Colin/Fehr, Ernst/Gintis, Herbert/McElreath, Richard, 2001: In Search of Homo Economicus: Behavioral Experiments in 15 SmallScale Societies, in: American Economic Review 91, 73-78.

Herrmann, Benedikt/Thöni, Christian/Gächter, Simon, 2008. Antisocial Punishment Across Societies, in: Science 319, 1362-1367.

Homans, George C., 1958: Human Behavior as Exchange, in: American Journal of Sociology 63, 597-606. 
Homans, George C., 1961: Social Behavior. Its Elementary Forms. Harcourt, Brace and World. Ioannidis, John P. A., 2005. Why Most Published Research Findings are False, in: PLoS Medicine 2, 696-701.

Kagel, John H./Roth, Alvin E. (Hrsg.), 1995: Handbook of Experimental Economics. Princeton, N.J.: Princeton University Press.

Ledyard, John O., 1995: Public Goods. A Survey of Experimental Research, in: Kagel, John H./Roth, Alvin E. (Hrsg.), Handbook of Experimental Economics. Princeton, N.J.: Princeton University Press, 111-194.

McAdams, Richard H., 2000. Signaling Discount Rates: Law, Norms, and Economic Methodology, in: The Yale Law Journal 110, 625-689.

McCabe, Kevin A./Rigdon, Mary L./Smith, Vernon L., 2003: Positive Reciprocity and Intentions in Trust Games, in: Journal of Economic Behavior and Organization 52, 267-275.

Malinowski, Bronislaw, 1926: Crime and Custom in Savage Society. London: Routledge and Kegan.

Mauss, Marcel, 1990 [1923/29]: The Gift. The Form and Reason for Exchange in Archaic Society. London: Routledge.

Meier, Stephan, 2006: A Survey of Economic Theories and Field-Evidence on Pro-Social Behavior. Working paper No. 06-6 der Federal Reserve Bank of Boston, Research Center for Behavioral Economics and Decision Making.

Ockenfels, Axel, 1999: Fairneß, Reziprozität und Eigennutz. Tübingen: Mohr-Siebeck.

Oosterbeek, Hessel/Sloof, Randolph/van de Kuilen, Gijs, 2003: Cultural Differences in Ultimatum Game Experiments: Evidence From a Meta-Analysis. Department of Economics, University of Amsterdam: Mimeo.

Preisendörfer, Peter, 1995: Vertrauen als soziologische Kategorie, in: Zeitschrift für Soziologie 24, 263-272.

Popitz, Heinrich, 1961: Soziale Normen, in: Europäisches Archiv für Soziologie 2, 185-198.

Posner, Eric A., 2000: Law and Social Norms. Cambridge, Mass: Harvard University Press.

Prosch, Bernhard, 2007. Kooperation durch soziale Einbettung und Strukturveränderung. Ergebnisse eines Forschungsprogramms experimenteller Spiele. Habilitationsschrift an der Wirtschafts- und Sozialwissenschaftlichen Fakultät der Universität Erlangen-Nürnberg.

Rabin, Matthew, 1993: Incorporating Fairness into Game Theory and Economics, in: American Economic Review 83, 1281-1302.

Rapoport, Anatol, 1998. Decision Theory and Decision Behaviour. London: Macmillan.

Rapoport, Anatol/Chammah, Albert M., 1965: Prisoner's Dilemma. Ann Arbor: University of Michigan Press.

Raub, Werner, 1992: Eine Notiz über die Stabilisierung von Vertrauen durch eine Mischung von wiederholten Interaktionen und glaubwürdigen Festlegungen, in: Analyse und Kritik 14, 187-194.

Raub, Werner/Buskens, Vincent, 2006: Spieltheoretische Modellierungen und empirische Anwendungen in der Soziologie, in: Diekmann, Andreas (Hrsg.), Methoden der Sozialforschung. Sonderheft 44 der Kölner Zeitschrift für Soziologie und Sozialpsychologie. Wiesbaden: VSVerlag, 560-598.

Raub, Werner/Weesie, Jeroen, 1990: Reputation and Efficiency in Social Interactions. An Example of Network Effects, in: American Journal of Sociology 96, 626-654.

Regan, Dennis T. 1971. Effects of Favor and Liking on Compliance, in: Journal of Experimental Social Psychology 7, 627-639.

Rilling, James K./Gutman, David A./Zeh, Torsten R./Pagnoni, Giuseppe/Berns, Gregory S./Kilts, Clinton D., 2002: A Neural Basis for Social Cooperation, in: Neuron 35, 395-405.

Selten, Reinhard, 1965: Spieltheoretische Behandlung eines Oligopolmodells mit Nachfrageträgheit. Teile I und II, in: Zeitschrift für die gesamte Staatswissenschaft 121, 301-324 und 667-689. 
Simmel, Georg, 1950: The Sociology of Georg Simmel. Edited and translated by K. H. Wolff. Glencoe: The Free Press.

Snijders, Chris, 1996. Trust and Commitments. Amsterdam. Thesis Publishers.

Sorokin, Pitirim A., 1928: Arbeitsleistung und Entlohnung (Experimentelle Untersuchungen bei Kindern im Alter von 3 - 4 Jahren und von 13 - 14 Jahren), in: Kölner Vierteljahreshefte für Soziologie 7, 186-198.

Taylor, Michael, 1976: Anarchy and Cooperation. New York: Wiley.

Titmuss, Richard M., 1970: The Gift Relationship. London: Allen und Unwin.

Thaler, Richard H., 1992: The Winner's Curse. Paradoxes and Anomalies of Economic Life. Princeton, N.J.: Princeton University Press.

Tutic, Andreas/Liebe, Ulf, 2008: A Theory of Status-Mediated Inequity Aversion. Arbeitspapier Universitat Leipzig.

Tversky, Amos/Kahneman, Daniel, 1981: The Framing of Decisions and the Psychology of Choice, in: Science 211, 453-458.

Vieth, Manuela/Weesie, Jeroen, 2007. Trust and Promises as Friendly Advances. Experimental Evidence on Reciprocated Kindness. University of Utrecht: ICS working paper.

Villeval, Marie-Claire, 2007. Experimental Economics. Contributions, Recent Developments, and New Challenges. University of Lyon: GATE Groupe d'Analyse et de Théorie Économique.

Voss, Thomas, 1998: Strategische Rationalität und die Realisierung sozialer Normen, in: Müller, Hans-Peter/Schmid, Michael (Hrsg.), Norm, Herrschaft und Vertrauen. Opladen: Westdeutscher Verlag, 117-135.

Voss, Thomas, 2001: Game Theoretical Perspectives on the Emergence of Social Norms, in: Hechter, Michael/Opp, Karl-Dieter (Hrsg.), Social Norms. New York: Russell Sage, 105-136.

Webb, E. J./Campbell. D. T./Schwartz, R. D./Sechrest, R., 1966: Unobtrusive Measures. Nonreactive Research in the Social Sciences. London: Sage.

Weesie, Jeroen/Raub, Werner, 1996. Private Ordering. A Comparative Institutional Analysis of Hostage Games, in: Journal of Mathematical Sociology 21, 201-240.

Weimann, Joachim, 1994: Individual Behaviour in a Free Riding Experiment, in: Journal of Public Economics 54, 185-200.

Xiao, Erte/Bicchieri, Cristina, 2008: When Equality Trumps Reciprocity: Evidence From a Laboratory Experiment. MPRA-Arbeitspapier 9375: Universität München.

Yang, Chung-Lei/Weimann, Joachim/Mitropoulos, Atanasios, 2006: An Alternative Approach to Explaining Bargaining Behaviour in Simple Sequential Games, in: Pacific Economic Review $11,201-221$.

Diekmann, Andreas, 1951, Prof. Dr., Professor für Soziologie an der ETH Zürich. Forschungsgebiete: Umweltsoziologie, Bevölkerung, Methoden und Modelle, Experimentelle Spieltheorie und Untersuchungen zur sozialen Kooperation. Veröffentlichungen: The Wealth of Nations and Environmental Concern, Environment and Behavior 31, 1999 (mit A. Franzen); The Social Inheritance of Divorce, American Sociological Review 64, 1999 (mit H. Engelhardt); Umweltsoziologie. Eine Einführung, Reinbek 2001 (mit P. Preisendörfer); Vertrauen und Reputation bei Internet-Auktionen, in: Kölner Zeitschrift für Soziologie und Sozialpsychologie 54, 2002 (mit D. Wyder); Do Parents of Girls Have a Higher Risk of Divorce? Journal of Marriage and the Family 66, 2004 (mit K. Schmidheiny); The Power of Reciprocity. Journal of Conflict Resolution 48, 2004; Methoden der Sozialforschung (Hrsg.): Sonderheft 44 der Kölner Zeitschrift für Soziologie und Sozialpsychologie, Wiesbaden 2006; Empirische So-zialforschung, Reinbek 2007; Not the First Digit! Using Benford's Law to Detect Fraudulent Scientific Data. J. of Applied Statistics 34, 2007: 321-329; Spieltheorie. Eine Einführung, Reinbek (in Vorbereitung). 